\title{
Calculation of Hydraulic System of Movable Rigid Barrier Crawler Track
}

\author{
Wang Huaiyu, Song Jianfeng \\ Tianjin University of Technology and Education, school of automobile and \\ transportation, 300222,China
}

\begin{abstract}
The load, speed and working requirements of a hydraulic system are analyzed and the load of its walking mechanism is calculated according to the composition of the hydraulic system of a movable rigid barrier crawler track, so the related parameters of the main components of the hydraulic system can be obtained. The type of the hydraulic components and driving components is selected according to the calculation results to satisfy the working requirements of the hydraulic system. Finally the type selection results are validated by verifying motion of the key function components of the hydraulic system, so it lays the foundation for system engineering implementation.
\end{abstract}

KEYWORDS: Rigid barrier; crawler track; Calculation of hydraulic system

\section{Introduction}

The movable rigid barrier crawler track is composed of a mechanical system, a hydraulic system, a control system and an energy system to move rigid barriers in car collision test, adapt to quick replacement and maintenance of rigid barriers, and secure operators. [1]

Its mechanical system mainly supports car body and move an object. The hydraulic system transfers power and motion and is composed of different hydraulic elements and hydraulic oils. The control system controls and regulates operation of the hydraulic system and is composed of PLC, frequency transformer and radio receiver. The energy system stores and converts power and is composed of battery and AC/DC converter.

The hydraulic system includes the power elements and executive elements, is first calculated in components of the crawler track, and is the prerequisite of other designs. 


\section{Composition of hydraulic system of movable rigid barrier crawler track}

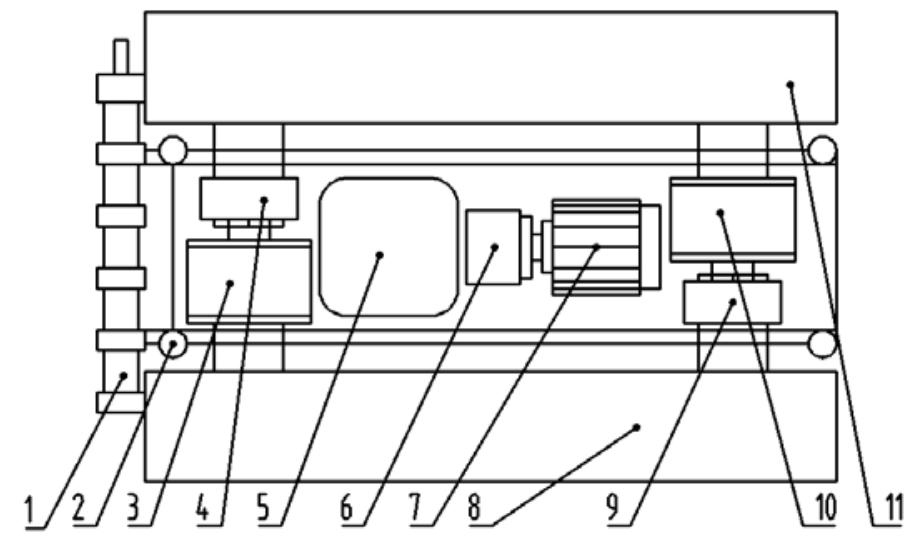

Fig. 1 Composition of movable rigid barrier crawler track

The hydraulic system of the movable rigid barrier crawler track is composed of the hydraulic oil source system, hoisting mechanism and walking mechanism.[2] The hydraulic oil source system is composed of the oil tank 5, hydraulic pump 6 and motor 7. The hoisting mechanism is composed of synchronous hydraulic cylinder 1 and four piston hydraulic cylinder 2. The walking mechanism includes two hydraulic motors 4 and 9 as the executive element, which will respectively drive the reduction gearbox 3 , track 8 , reduction gearbox 10 and track 11 in the mechanical system.

The hoisting mechanism is interlocked with the walking mechanism. The hydraulic oil source system cannot provide two systems with the power simultaneously. When the hoisting mechanism fully ascends or descends and is locked, the walking mechanism can work.

\section{Load requirements of hydraulic system}

The movable rigid barrier crawler track can carry 100t and the dead weight is $15 \mathrm{t}$. The speed is about $0.1 \mathrm{~m} / \mathrm{s}$. The crawler track can rotate at the center and scrap edge. The ascending and descending rate of the platform is $5 \mathrm{~mm} / \mathrm{s}$. The platform runs stably and the positioning precision of the walking mechanism is common.

The hydraulic system of the crawler track is used for two purposes. First, the hydraulic motor transfers the torsion to the track via the reduction gearbox. Each motor only drives a track. Driving status of the crawler track can be controlled by controlling the operation status of the motor. Second, the hoisting mechanism makes the rigid barrier ascend and descend. The hydraulic oil will be uniformly assigned to 
four piston cylinders via the synchronous hydraulic cylinders and hoist the rigid barrier.

\section{Working principle of hydraulic system}

The open oil source loop system is used by the hydraulic system of the crawler track. For its principle diagram, refer to the figure 2.

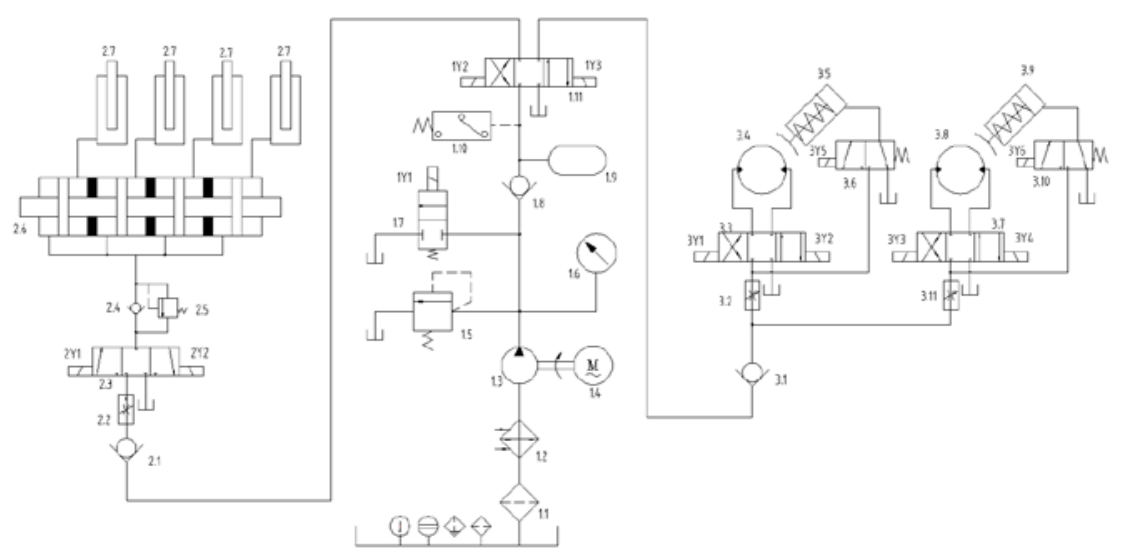

Fig. 2 Principle drawing of hydraulic system

The hydraulic oil flows to the hydraulic pump 1.3 driven by the motor 1.4 via the filter 1.1 and the cooler 1.2. The pressure meter 1.6 is observed to regulate the overflow valve 1.5 and control the pressure supplied by the hydraulic oil source. The one-way valve 1.8 can prevent the hydraulic oil from returning.

The hoisting mechanism is remotely controlled in operation. The three-position and four-way solenoid valve 1.11 will trigger $1 \mathrm{Y} 3$ and three-position and three-way solenoid valve 2.3 triggers $2 \mathrm{Y} 1$. The walking mechanism is locked and cannot work. The one-way valve 3.1 can prevent the hydraulic oil of the walking mechanism from returning to the oil tank. The hydraulic oil can stabilize the pressure via the speed control valve 2.2 to synchronize the hydraulic cylinder 2.6 and make four piston cylinders 2.7 ascend synchronously. After the platform ascends to the top, the solenoid valve 1.11 will be disconnected from 2.3 and return to the position $\mathrm{O}$ and the piston cylinder 2.7 stops operation. The pressure relay 1.10 triggers $1 \mathrm{Y} 1$ and connects the solenoid valve 1.7 for unloading and pressure reduction. After the hydraulic pump stops operation, the relay 1.10 and the solenoid valve 1.7 reset.

The walking mechanism is remotely controlled for operation and the solenoid valve 1.11 triggers 1 Y2. The hoisting mechanism is locked and cannot operate. Oneway 2.1 prevent the hydraulic oil of the hoisting mechanism from returning to the oil tank. The speed control valve 3.2 and 3.11 will regulate the flow to ensure that the

\section{Published by Francis Academic Press, UK}


rotation speed of two hydraulic motors is same. The walking mechanism can forward, backward and rotate by controlling the operation position of three-position and four-way solenoid valve 3.3 and 3.7. The two-position and three-way valve 3.6 and 3.10 can be remotely controlled to break the walking mechanism via the embedded brake 3.5 and 3.9 inside the hydraulic motor in walking.

After the crawler track reaches the transport position, the solenoid valve 1.11 returns to the position $\mathrm{O}$ and the hoisting mechanism and the walking mechanism will stop operation. The solenoid valve 2.3 triggers $2 \mathrm{Y} 2$ and the hoisting mechanism ascends. One-way valve 2.4 and the overflow valve 2.5 compose a balance loop to make the hydraulic oil return to the oil tank and generate the back pressure. The piston cylinder will slowly descend to the lowest end and the crawler track completes operation.

\section{Calculation of hydraulic system}

The rigid barrier is a steel reinforced concrete cuboid and its dimension is $4.6 \mathrm{~m} \times 4.6 \mathrm{~m} \times 2 \mathrm{~m}$. A gap is under the rigid barrier for the crawler track to move out and in and its dimension $1.05 \mathrm{~m} \times 2.55 \mathrm{~m} \times 4.3 \mathrm{~m} .{ }^{[3]}$ To enhance the collision resistance, the rigid barrier is made of the heavy concrete and its density is about $3300 \mathrm{~kg} / \mathrm{m}^{3}{ }^{[4]}$ The barrier mass is expressed as

$$
m_{b}=V_{b} \rho=(4.6 \times 4.6 \times 2-1.05 \times 2.55 \times 4.3) \times 3300=101.67 t .
$$

The deviation of the calculated from the designed load error is less than $2 \%$, so the data are valid.

\subsection{Calculation of hydraulic system of the walking mechanism}

The transport quality of the walking mechanism is the sum of the barrier and crawler track. Given $K=1.2$, we can get $m^{\prime}=(101.67+15) \times 1.2=140 t$. The driving resistance coefficient is expressed as $f=0.04$, the theoretical steering resistance coefficient is expressed as $\mu_{i}=0.18$, the radius of the driving wheel is expressed as $r_{d q}=0.45 \mathrm{~m}$, the track length is expressed as $L=3 \mathrm{~m}$, the track gauge is expressed as $B=1.3 m$ and the track width is expressed as $b=0.6 m$.

\subsubsection{Analysis on hydraulic motor load}

The steering resistance coefficient is 4.5 time of the driving resistance coefficient, so the load of the hydraulic motor is calculated according to the resistance of the crawler track in steering.

When the gravity center of the crawler track does not deviate, the resistance equation of single track is expressed as $F_{q \max }=\frac{G}{2} \times\left(f+\frac{\mu L}{2 B}\right)$.

\section{Published by Francis Academic Press, UK}


When the gravity center deviates, the gravity center deviates to the track on one side and the resistance equation is:

$$
F_{q}^{\prime}=\frac{f G}{2}\left(1+\frac{2 C}{B}\right)+\frac{\mu G L}{4 B}\left[1-\left(\frac{2 e}{L}\right)^{2}\right]^{2}
$$

When the lateral eccentricity is expressed as $C=\frac{B}{2}$ and longitudinal eccentricity is expressed as $e=0$, the driving toque of the track close to the eccentric side is maximal. These parameters are substituted to the above equation to get $F_{q \max }^{\prime}=\frac{G}{2} \times\left(2 f+\frac{\mu L}{2 B}\right)$.

Namely $\frac{F_{q \max }^{\prime}}{F_{q \max }}=1.16<K=1.2$, it indicates that the proportion of the increased distance due to gravity center deviation is within the set security coefficient, the deviation of the gravity center of the crawler track is not considered in calculation.

From the experience equation of the real steering resistance coefficient $\mu=\frac{\mu_{i}}{0.85+0.15 \frac{R}{B}}$, when the steering radius (R) of the crawler track is 0 (center steering), the steering resistance coefficient is maximal, namely $\mu=0.212$ and the maximal load of single track of the crawler track is:

$$
F_{q \max }=\frac{G}{2} \times\left(f+\frac{\mu L}{2 B}\right)=\frac{140000 \times 9.8}{2} \times\left(0.04+\frac{0.212 \times 3}{2 \times 1.3}\right)=195060 \mathrm{~N}
$$

\subsubsection{Calculation of hydraulic motor parameters}

The medium pressure grade is used as the operation pressure of the hydraulic motor, namely $p_{1}=16 \mathrm{MPa}$. The torque provided by the driving wheel shall be more than:

$$
M_{\max }=F_{q \max } r_{d q}=195060 \times 0.45=87777 N \cdot M
$$

The decelerator with the nominal transmission ratio $i=20$ is used, the torque of the hydraulic motor is expressed as $M_{k}{ }^{\prime}=4389 \mathrm{~N} \cdot \mathrm{m}$. The radial bulb stopper motor with $3 Q J M 32-2.0 S e$ is selected. The discharge capacity is divided into $2.030 \mathrm{~L} / \mathrm{r}$, $1.015 \mathrm{~L} / \mathrm{r}$ and $0.508 \mathrm{~L} / \mathrm{r}$. The rated torque is also divided into $4807 \mathrm{~N} \cdot \mathrm{M}$, $2404 N \cdot M$ and $1202 N \cdot M$ class according to the discharge capacity. The maximal pressure is $25 \mathrm{MPa}$. The continuous rotation range is $2 \sim 200 \mathrm{r} / \mathrm{min}$ and the mass is $120 \mathrm{~kg}$. The externally controlled brake is provided. The brake moment is $4000 N \cdot M$, the real capacity efficiency is 0.95 and the mechanical efficiency is 0.92 . 
The discharge capacity of the hydraulic motor is $2.030 \mathrm{~L} / \mathrm{r}$ in case of steering and the output torque is increased. The pilot valve changes the position the speed control valve inside the motor in normal straight driving to make the discharge capacity of the hydraulic motor reach $0.508 \mathrm{~L} / \mathrm{r}$, reduce the output torque, and increase the speed.

\subsubsection{Calculation of hydraulic pump parameters}

(1) Calculation of maximal output pressure of hydraulic pump

The maximal operation pressure of the hydraulic pump is identified according to the experience equation, namely $p_{p} \geq p_{1}+\sum \Delta p=16+1.5=17.5 \mathrm{MPa}$.

Wherein $p_{1} \_$Operation pressure of the hydraulic cylinder, $\mathrm{MPa}$;

$\sum \Delta p \longrightarrow$ Total pipeline loss, $1.5 \mathrm{MPa}$ is taken.

The hydraulic pump shall reserve certain pressure. The maximal output pressure of the pump is $25 \% \sim 60 \%$ higher than the maximal operation pressure. The range of the maximal output pressure of the hydraulic pump calculated based on $p_{p} \geq 17.5 \mathrm{MPa}$ is $21.875 \mathrm{MPa} \leq p_{p 0} \leq 28 \mathrm{MPa}$.

(2) Calculation of hydraulic pump flow

Given that the preset vehicle rate is $v_{Y}=0.1 \mathrm{~m} / \mathrm{s}$, the rated rotation speed of the driving wheel is calculated as follows:

$$
n_{Y}=\frac{v_{Y}}{2 \pi r_{d q}}=\frac{0.1}{2 \pi \times 0.45} \times 60=2.122 r / \min
$$

The preset rotation rate of the hydraulic motor is expressed as $n^{\prime}=n_{Y} i=2.122 \times 20=42.44 \mathrm{r} / \mathrm{min}$.

The input flow of the hydraulic motor is expressed as $q^{\prime}=\frac{V^{\prime} n^{\prime}}{\eta_{V}^{\prime}}=\frac{0.508 \times 42.44}{0.95}=22.694 \mathrm{~L} / \mathrm{min}$.

Wherein: $V^{\prime}$-- discharge capacity of the hydraulic motor, $L / \mathrm{min}$

$n^{\prime}$ —expected rotation rate of hydraulic motor, $r / \mathrm{min}$;

$\eta_{V}^{\prime} \longrightarrow$ motor efficiency of the hydraulic motor, 0.95 is taken.

Two hydraulic motors will operate simultaneously and the output flow of the hydraulic pump is: ${ }^{[5]}$

$$
q_{p}^{\prime} \geq K \sum q_{\text {max }}^{\prime}=1.3 \times(22.694 \times 2+3)=62.904 \mathrm{~L} / \mathrm{min}
$$


Wherein: $q_{p}^{\prime}{ }_{p}$ flow of the hydraulic pump in the hydraulic system of the walking mechanism, $L / \min$;

$\mathrm{K} —$ system leakage coefficient, 1.3 is taken;

$\sum q_{\max }^{\prime}$ maximal flow of simultaneously operating hydraulic motors plus the minimal overflows $3 \mathrm{~L} / \mathrm{min}$ of the overflow valve.

(3) Driving power of hydraulic pump

If no burst acceleration occurs and the hydraulic pump pressure and flow are constant, the minimal driving power of the hydraulic pump is calculated as follows: ${ }^{[6]}$

$$
P^{\prime}=\frac{p_{p}^{\prime} q_{p}^{\prime}}{60 \eta_{p}^{\prime}}=\frac{17.5 \times 62.904}{60 \times 0.7}=26.210 \mathrm{~kW}
$$

Wherein: $p_{p}^{\prime}$ — maximal operation pressure of the hydraulic pump, $\mathrm{MPa}$;

$q_{p}^{\prime}$ — Flow of hydraulic pump, $L / \mathrm{min}$;

$\eta_{p}^{\prime}{ }_{p}$ Total efficiency of hydraulic pump, 0.7 is taken.

\subsection{Selection of hydraulic pump and driving motor}

The parameters of the hydraulic pump and driving motor are calculated and summarized according to the above equations:

(1) Range of maximal output pressure of the hydraulic pump: 21.875MPa $\leq p_{p 0} \leq 28 M P a$

(2) Minimal flow of the hydraulic pump of the walking mechanism is $62.904 \mathrm{~L} / \mathrm{min}$.

(3) The minimal power of the driving motor of the walking mechanism is $26.210 \mathrm{~kW}$.

$P V 2 R 2$ - 47 Blade pump is selected, the discharge capacity is $47 \mathrm{~mL} / \mathrm{r}$ and the rotation speed is $600 \sim 1800 \mathrm{r} / \mathrm{min}$ and the rated pressure is $21 \mathrm{MPa}$ according to the requirements. 


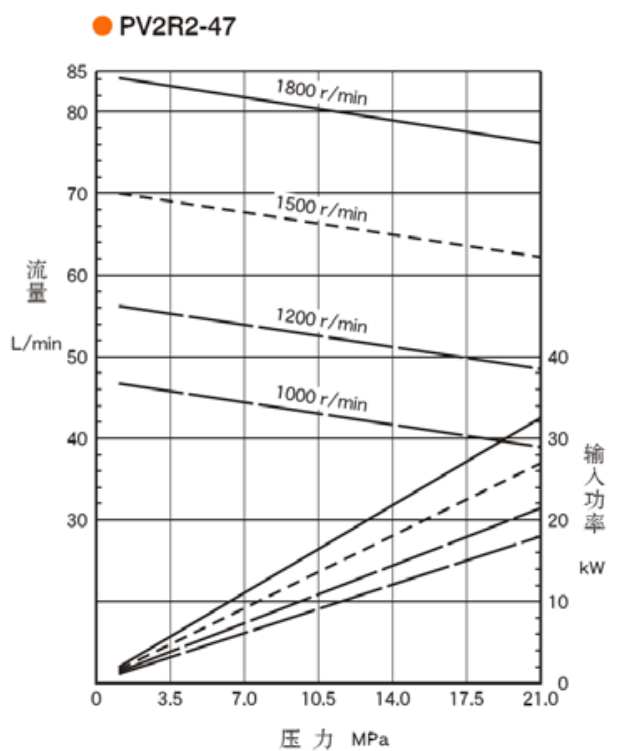

Fig. 3 Pressure, flow and input power characteristics of PV2R2-47 hydraulic pump pressure

The motors are selected by referring to "Design manual of mechanical engineers" according to the figure 3 .

Table 1 Motor parameters

\begin{tabular}{|c|c|c|c|c|c|c|}
\hline Model & $\begin{array}{c}\text { Rated } \\
\text { power } \\
k W\end{array}$ & $\begin{array}{c}\text { Rated } \\
\text { current } \\
\mathrm{A}\end{array}$ & $\begin{array}{c}\text { Rotation } \\
\text { rate } \\
\mathrm{r} / \mathrm{min}\end{array}$ & $\begin{array}{c}\text { Efficiency } \\
\%\end{array}$ & $\begin{array}{c}\text { Power } \\
\text { factor } \\
\cos \phi\end{array}$ & $\begin{array}{c}\text { Mass } \\
\mathrm{kg}\end{array}$ \\
\hline Y200L-4 & 30 & 56.8 & 1470 & 92.2 & 0.87 & 270 \\
\hline
\end{tabular}

\subsection{Check of hydraulic system}

5.3.1 Analysis on hydraulic cylinder load

The security coefficient $K=1.2$ is used for calculation, namely $m=101.67 \times 1.2=122 t$. The force is analyzed based on it and the maximal total load is calculated. 
When the rigid barrier ascends, it only touches the hydraulic cylinder and no friction resistance exists. The ascending speed of the platform is $5 \mathrm{~mm} / \mathrm{s}$ to make the inertial force less than $1 \%$ of the load force. The inertial force is ignored in calculation.

The gravity of the rigid barrier on the hydraulic cylinder is the main work load of the liquid of the hoisting mechanism liquid. The rigid barrier is hoisted by four hydraulic cylinders. One plane is identified by using three points not in a line, so three hydraulic cylinders are used as the benchmark in calculation.

$$
\text { Load force } F_{W}=F_{g}=\frac{m g}{3}=112 \times 10^{3} \times 9.8 \div 3=365867 \mathrm{~N}
$$

Given that the mechanical efficiency of the hydraulic cylinder is expressed as $\eta_{m}=0.9$, the hydraulic cylinder has no friction resistance on the startup phase and $F_{1}=0 N$. No touch occurs in case of acceleration due to the distance between the hydraulic cylinder and the rigid barrier and $F_{2}=0 N$. The load force on the constant speed stage:

$$
F_{3}=\frac{F_{W}}{\eta_{m}}=\frac{365867}{0.9}=406.519 \mathrm{kN}
$$

\subsubsection{Calculation of hydraulic cylinder parameter}

The speed control valve is used to control the pressure. The operation pressure is set as $p_{1}=16 \mathrm{MPa}$.

$$
\begin{aligned}
& \text { The working chamber area of the piston cylinder is } \\
& A=\frac{F_{3}}{p_{1}}=\frac{406519}{16 \times 10^{6}}=0.0254074 \mathrm{~m}^{2}=254.074 \mathrm{~cm}^{2} \text {. } \\
& \text { The inner diameter of the hydraulic cylinder is expressed as }
\end{aligned}
$$
$D=\sqrt{\frac{4 A}{\pi}}=\sqrt{\frac{4 \times 254.074}{\pi}}=17.986 \mathrm{~cm}$ and is rounded. $180 \mathrm{~mm}$ is selected. ZG-E180 single- acting piston cylinder is selected according to the above information, the stroke is $200 \mathrm{~mm}$, the rated pressure $16 \mathrm{MPa}$, the maximal push is $430 \mathrm{kN}$ and the maximum stroke $4000 \mathrm{~mm}$.

\subsubsection{Parameter calibration of hydraulic pump}

(1) Pressure calibration of hydraulic pump

The operation pressure of the hydraulic cylinder is expressed as $p_{1}=16 \mathrm{MPa}$ and the experience equation used by the operation pressure of the hydraulic pump is same, so the maximal output pressure range of the hydraulic pump is same and is expressed as $21.875 \mathrm{MPa} \leq p_{p 0} \leq 28 \mathrm{MPa}$, it complies with the conditions.

\section{Published by Francis Academic Press, UK}


(2) Flow calibration of hydraulic pump

The piston stroke is $200 \mathrm{~mm}$ and the rate is $5 \mathrm{~mm} / \mathrm{s}$ according to the design requirements, the flow required by single piston cylinder is calculated as follows:

$\mathrm{q}_{i}=A v=\pi \times 90^{2} \times 5=127235 \mathrm{~mm}^{3} / \mathrm{s}=0.127235 \mathrm{~L} / \mathrm{s}=7.634 \mathrm{~L} / \mathrm{min}(\mathrm{i}=1,2,3,4)$

The output flow of the hydraulic pump is expressed as $q_{p} \geq K \sum q_{\max }=1.3 \times(7.634 \times 4+3)=43.597 \mathrm{~L} / \mathrm{min}$

Wherein: $\mathrm{K} —$ system leakage coefficient, 1.3 is taken.

$\sum q_{\max } \longrightarrow$ maximal flow of simultaneously acting hydraulic executive elements plus the minimal overflow $(3 \mathrm{~L} / \mathrm{min}$ ) of the overflow valve.

The calculated output flow of the hydraulic pump is $43.597 \mathrm{~L} / \mathrm{min}$, which is lower than the work flow $63 \mathrm{~L} / \mathrm{min}$ of the hydraulic pump. It complies with the conditions.

(3) Calibration of driving power of the hydraulic pump

If no burst acceleration occurs and the hydraulic pump pressure and flow are constant, the minimal driving power of the hydraulic pump is calculated as follows:

$$
P=\frac{p_{p} q_{p}}{60 \eta_{p}}=\frac{17.5 \times 43.597}{60 \times 0.7}=18.166 k W
$$

Wherein: $p_{p} \_$maximal operation pressure of hydraulic pump, $M P a$;

$q_{p}$-flow of hydraulic pump, $L / \mathrm{min}$;

$\eta_{p} \_$total power of hydraulic pump, 0.7 is taken.

The calculated minimal driving power of the hydraulic pump is $18.166 \mathrm{~kW}$, which is lower than the output power $27.66 \mathrm{~kW}$ of the motor. It complies with the conditions.

\subsection{Speed calibration}

The estimated work flow is $63 \mathrm{~L} / \mathrm{min}$ under the $1470 \mathrm{r} / \mathrm{min}$ rotation rate and 17.5 $\mathrm{MPa}$ operation pressure according to the figure 3 . It is substituted to the flow calculation equation $\left(q_{p}=1.3 \times(2 q "+3)\right.$ ) of the hydraulic pump to get the input work flow $(q "=22.731 \mathrm{~L} / \mathrm{min})$ of the hydraulic motor.

Operation rotation rate of the hydraulic motor is expressed as $n^{\prime \prime}=\frac{q " \eta_{V}^{\prime}}{V^{\prime}}=\frac{22.731 \times 0.95}{0.508}=42.508 \mathrm{r} / \mathrm{min}$; 
The operation rate of the crawler track is expressed as $v_{e}=\frac{2 \pi r_{d q} n^{\prime \prime}}{60 i}=\frac{2 \pi \times 0.45 \times 42.508}{60 \times 20}=0.100 \mathrm{~m} / \mathrm{s}$ the obtained result approximates to the preset rate $0.1 \mathrm{~m} / \mathrm{s}$. It complies with the conditions.

\section{Conclusions}

The model of the hydraulic cylinder, hydraulic motor, hydraulic pump and driving motor is properly selected in the above calculation and the hydraulic system of the crawler track is calculated for validation. This hydraulic system can satisfy the basic requirements of the movable rigid barrier and one driving motor can drive the hydraulic pump to make the hoisting mechanism and walking mechanism of the crawler track operate in different intervals. After computation and calibration, the selected models are reasonable and the calculated hydraulic system of the crawler track of the movable rigid barrier is feasible.

\section{References}

[1] DSD (2015). Operating and Maintenance Instructions Crawler track for crashblock. Germany: Bargfrieder.

[2] Liu Yuguang, Liu Zhinxin (2015)..Current Conditions and Development of Test Evaluation Technologies In New Vehicle Evaluation Regulations (Ncap) of Different Countries . Car Safety and Energy Saving Journal, 2013, Volume, issue 1: $016-022$

[3] Wu Huige, Chen Huiqin(2011). Research And Engineering Application of Heavy Concrete. Concrete, No. 11, 107-109

[4] Li Zhigang, Gong Haixi (2015). Simulation Analysis of Single-Pump Driven Double-Hydraulic Motor System. Machine Tool and Hydraulics, Vol. 43, No. 17: 157-159

[5] Han Guihua, Shi Xianyu and Fan Chunbo (2014). Design Skills and Taboos of Hydraulic System. Version 2, Beijing: Chemistry Industry Press. 